\title{
Nouvelles du domaine Tarifs et Conventions
}

\section{Ernst Gähler ${ }^{a}$ Irène Marty ${ }^{b}$}

a Vice-président de la FMH, responsable du domaine Tarifs et Conventions

b Cheffe des Tarifs et Conventions

\section{TARMED Suisse}

Le 15 septembre 2010, le comité de direction de TARMED Suisse a décidé que, pour l'instant, il n'enverrait pas la nouvelle version 1.08 du TARMED au Conseil fédéral pour approbation car les réflexions concernant le rapport coûts-utilité sont défavorables. L'introduction de la nouvelle version est donc repoussée d'un an et elle entrera probablement en vigueur le $1^{\text {er }}$ mars 2012.

\section{Contrôle et renvois de factures par quelques caisses}

Ces dernières semaines, le domaine Tarifs et Conventions a observé une nouvelle recrudescence des problèmes rencontrés par de nombreux médecins au sujet du contrôle et du renvoi de factures par quelques caisses-maladie. Celles-ci informent souvent aussi simultanément les patients, ce qui provoque une certaine insécurité.

Il s'agit souvent de problèmes autour de la valeur intrinsèque (prestations dans le domaine des droits acquis) ou de la reconnaissance des unités fonctionnelles (comme la salle d'opération au cabinet ou la psychothérapie déléguée). Vous trouverez dans le Bulletin des médecins suisses [1] des informations concernant la marche exacte à suivre dans de tels cas et comment procéder.

Nous avons aussi constaté de «nouvelles apparitions». Nous vous présentons ci-après nos commentaires sur les nouvelles raisons les plus importantes invoquées par quelques caisses lorsqu'elles renvoient des factures:

\section{Interdiction de cumuler des prestations «le même jour»}

Une caisse effectue systématiquement des renvois, générés par son programme informatique, de positions tarifaires avec l'interdiction de cumul («non cumulable avec») qui sont facturées à la même date, toutefois pour différentes séances. Un exemple concret est la position tarifaire 00.0110 «Consultation téléphonique par le spécialiste» et la position 00.0060 «Visite». Le médecin a fourni ces deux prestations au patient le même jour mais à des moments différents, et il les a donc aussi notées correctement sur la facture en mentionnant deux séances différentes.

Dans le navigateur TARMED sous «Remarques générales relatives au tarif», il est clairement dit qu'une telle interdiction de cumul entre deux presta- tions se réfère toujours à une séance [2]. La notion de séance est définie dans l'IG-8 [3]. La séance est l'unité de temps ayant servi de base au calcul des positions tarifaires dans les modèles de coûts du TARMED.

La facturation des positions 00.0110 «Consultation téléphonique par le spécialiste» et la position 00.0060 «Visite» est possible le même jour, la consultation téléphonique ayant eu lieu le matin et la visite chez le patient l'après-midi.

\section{Limitation de la position tarifaire 39.0010:}

«1 fois par séance» égale «1 fois par jour»

Un autre exemple concerne les radiologues en pratique libérale. La suppression de la décision CPI I-08051-B le $1^{\text {er }}$ avril 2010 a fait disparaître la limitation des positions tarifaires 39.0010/11 «Consultation de base/Unité d'exploitation Institut de radiologie en dehors de l'hôpital» de «1 fois par unité d'exploitation et 2 fois par jour». De cette façon, c'est à nouveau la limitation initiale qui figure dans la version 01.08 TARMED pour ces positions, à savoir «1 fois par séance».

Une caisse refuse maintenant des factures contenant cette prestation 2 fois par jour au motif qu'avec la suppression de la décision CPI, cette prestation ne serait admise plus qu' 1 fois par jour. C'est naturellement faux car les positions 39.0010/11 se réfèrent maintenant à nouveau à chaque séance en particulier et non pas à une journée. De ce fait, elles peuvent être facturées plusieurs fois le même jour lorsque les prestations ont été fournies dans des séances différentes (différentes unités fonctionnelles).

Les médecins facturant des prestations de droits acquis sont à mettre sur un pied d'égalité avec les «spécialistes»

Une caisse a prétendu qu'un médecin facturant une prestation de droits acquis devait être mis sur un pied d'égalité pour cette prestation avec un spécialiste et que, par conséquent, il fallait appliquer, pour ces positions tarifaires, les règles prévues pour les spécialistes. Un exemple concret: lorsqu'ils facturent les positions du chapitre 39 «Imagerie médicale», les thérapeutes de la douleur n'auraient pas le droit de facturer d'autres positions. Cette règle est applicable aux spécialistes en radiologie médicale/radiodiagnostic (cf. IC-39-9 Bloc de prestations BP-53) [4], toutefois pas aux autres spécialistes. Cette règle a été limitée aux spécialistes en radiologie médicale/radiodiagnostic pour permettre aux non-radiologues (par exemple 
les médecins de famille) de fournir au patient des prestations d'imagerie conjointement avec d'autres examens durant la même séance. Si l'argumentation de cette caisse devait s'imposer sous cette forme, les patients devront bientôt s'armer d'un agenda consacré à leurs nombreuses consultations médicales.

\section{Conclusion}

Les caisses sont tenues de contrôler les factures et le corps médical admet aussi que ces contrôles soient effectués par les répondants des coûts.

Mais ce que nous voyons et entendons actuellement au domaine Tarifs et Conventions dépasse dans beaucoup de cas le contrôle correct d'une facture, n'est consigné dans aucun contrat, s'appuie sur des interprétations indéfendables et en partie complètement illogiques, provoque beaucoup de paperasserie administrative et d'irritations chez les médecins, et crée des doutes chez les patients. Sans parler des coûts occasionnés aux caisses maladie par ce genre de «contrôles erronés» que nous devons à nouveau financer en tant que payeurs de primes.

\section{Ce que nous voyons et entendons actuellement dépasse dans beau- coup de cas le contrôle correct d'une facture}

Il s'agit de mettre fin aux agissements de certaines caisses. Par notre ligne d'assistance téléphonique Infoline Tarifs 0900340 340, vous obtiendrez chaque lundi et chaque mercredi entre $9 \mathrm{~h}$ et $12 \mathrm{~h}$ des renseignements donnés par des personnes compétentes. Vous nous atteignez également par courriel à tarife@fmh.ch.

\section{Remise de médicaments par les médecins}

Le modèle FMH d'une indemnisation exempte de marge de la remise de médicaments par les médecins a donné lieu à une discussion avec une délégation de santésuisse en avril 2010. Lors de la séance du conseil d'administration tenue le 25 août 2010, santésuisse a décidé d'entrer en matière sur des négociations avec le corps médical. La FMH attend une proposition pour une première date de séance.

Le modèle FMH n'est pas un modèle d'économie mais il prévoit de transférer les rémunérations des médecins disposant de l'autorisation de remettre des médicaments sur des positions TARMED (PM et PT). Il est prévu de créer dans le TARMED une unité fonctionnelle «Remise de médicaments par le médecin» qui règle les conditions de la propharmacie, par exemple de manière analogue à l'unité fonctionnelle «Salle d'opération au cabinet médical». Nous ne pouvons pas tolérer que des économies soient à nouveau faites sur le dos des praticiens! Le nouveau système générera une augmentation de l'efficacité, laquelle ouvrira de nouvelles perspectives intéressantes aussi pour les assureurs-maladie.

\section{QUALAB: nouvelles valeurs de l'intervalle de tolérance dès le $1^{\mathrm{er}}$ janvier 2011}

La QUALAB a procédé à quelques adaptations de l'intervalle de tolérance concernant les contrôles de qualité internes et externes. Il s'agit des paramètres suivants: sodium, bilirubine totale, protéine totale, alanine-aminotransférase, amylase, hémoglobine glyquée (HbA1C), amylase pancréatique. Les nouvelles valeurs de l'intervalle de tolérance sont valables dès le $1^{\text {er }}$ janvier 2011.

\section{Références}

1 Gähler E, Marty I. Tarifs, conventions et une lettre à l'OFSP. Bull Méd Suisses. 2010;91(15):567-9.

2 TARMED: Remarques générales relatives au tarif:

Lors de la facturation de la position tarifaire, la prestation concernée ne peut pas être facturée conjointement avec les prestations/chapitres/groupes de prestations mentionnés sous «non cumulable avec» dans le cadre d'une seule et même séance.

3 IG-8 Séance:

Par «séance», on entend la période de temps (allant, dans le domaine ambulatoire, de la prise de contact avec le patient à son départ) durant laquelle un fournisseur de prestations est sollicité par un patient, un couple, une famille ou un groupe. Les séances manquées ne peuvent pas être portées à la charge des assurances sociales. La réanimation est considérée comme une séance séparée, sauf dans le cadre des soins intensifs et de l'anesthésie (BP-10 et BP-52).

4 IC-39-9 Bloc de prestations:

Pour les spécialistes en radiologie médicale/ radiodiagnostic, les positions tarifaires du chapitre 39 font partie d'un bloc de prestations et ne peuvent par conséquent être cumulées qu'entre elles (et donc avec aucune autre position du tarif) par le même spécialiste, pour une même séance. Font exception les positions tarifaires des suppléments pour consultation pressante ou urgence, sous-chapitre 00.08. Les autres exceptions sont expressément mentionnées. 